\title{
CARDIAC MR IMAGE SEGMENTATION: QUALITY ASSESSMENT OF STACS
}

\section{Charnchai Pluempitiwiriyawej and José M. F. Moura}

\author{
Electrical and Computer Engineering \\ Carnegie Mellon University \\ chanchai@ece.cmu.edu,moura@ece.cmu.edu
}

\author{
Pittsburgh NMR Center for Biomedical Research \\ Carnegie Mellon University
}

\begin{abstract}
We present an energy based automatic image segmentation algorithm that uses a novel active contour scheme, called the stochastic active contour scheme (STACS). The algorithm overcomes some unique challenges arising in cardiac magnetic resonance (MR) images by minimizing an energy functional with four terms, each representing the region and edge based information of the image and the global and local properties of the contour. We use annealing schedules to control the relative strength of each of the terms during the minimization process. The segmentation results when applying STACS to a set of real cardiac MR sequences of a rat are presented and quantitatively assessed by comparing them to the manually-traced contours using two similarity measures, the area and shape similarity measures. This assessment validates STACS's results, demonstrating its very good and consistent segmentation performance.
\end{abstract}

\section{INTRODUCTION}

Cardiac MR image segmentation is challenging because the texture of the myocardium (the heart muscle) is perceivably undistinguishable from the texture of other heart structures and anatomy parts, such as the papillary muscles or the chest wall. Most of the available active contour methods lead to problematic results when applied to cardiac MR images. Purely edge-based active contour schemes [1-3] are sensitive to noise and initialization, i.e., the initial contour must reside close enough to the true boundary for the contour to evolve. Further, turbulent blood flow in some cardiac MR images often causes faulty edges. Papillary muscles and other anatomy parts like the chest wall pose additional challenges for segmentation because they have textures similar to the myocardium's but should be excluded when segmenting the left ventricle. Purely region-based schemes [4,5], though more robust to initial conditions, can not resolve objects with similar textures, thus can not distinguish the myocardium from the papillary muscles or the chest wall. We address these problems by modeling the image textures stochastically and imposing prior shape constraint onto the contour. Our new active contour scheme for segmenting a cardiac MR image in small animal models (rats) is referred to as stochastic active scheme or STACS $[6,7]$. Our compounding energy functional includes stochastic modeling of the image textures and shape priors, in addition to the usual edge-based and contour smoothness terms. When applied to real MR image sequences of a rat's heart, STACS correctly segments the heart region (myocardium) from other anatomy parts.

This work was supported by NIH grants, R01EB/AI-00318 and P41EB001977, to Pittsburgh NMR Center for Biomedical Research, CMU.
In this paper, we describe an annealing algorithm on the parameters weighting the different terms of the STACS's energy functional and assess the quality of the STACS's segmentation results quantitatively with two similarity measures: area and shape. Section 2.1 summarizes STACS. In section 2.2, the annealing schedules are presented. We then validate the performance of STACS by comparing STACS's segmentation results to the ones obtained manually by an expert using the area similarity measure, described in section 3.1, and the shape similarity measure, detailed in section 3.2.

\section{STOCHASTIC ACTIVE CONTOUR}

\subsection{Energy Minimization}

We adopt an energy minimization approach to segment the heart and its structures (the left and right ventricles). Since MR images often have low contrast, it is very difficult to segment the myocardium from surrounding tissues, for example, the chest wall. A second major issue arises because the myocardium is essentially the same tissue as the papillary muscles but they are not to be included in the segmented left ventricle. To address successfully these problems, we design an energy functional that has four terms

$$
J(\mathbf{C})=\lambda_{1} J_{1}(\mathbf{C})+\lambda_{2} J_{2}(\mathbf{C})+\lambda_{3} J_{3}(\mathbf{C})+\lambda_{4} J_{4}(\mathbf{C}),
$$

where $\mathbf{C}$ is the contour that delineates the boundary of the desired heart structures (the left or right ventricle, or the epicardium), and $\lambda_{1}, \lambda_{2}, \lambda_{3}, \lambda_{4}$ are the relative weights of the four terms $J_{1}, J_{2}, J_{3}$, $J_{4}$, respectively. In the first term $J_{1}(\mathbf{C})$, we assume two different stochastic texture models: $M_{1}$ for the object (the left or right ventricle), residing within the contour, and $M_{2}$ for the background, residing outside the contour. This term is called the region-based term because it utilizes the regional statistics within the image to help evolving the contour $\mathbf{C}$ during the segmentation process. The second term $J_{2}(\mathbf{C})$ is the edge-based term. Its purpose is to attract the contour $\mathbf{C}$ to clues given by prominent edges within the edge map, derived from the image. The third term $J_{3}(\mathbf{C})$ regulates the global properties of the contour to resemble the prior knowledge about the heart shape $\mathbf{C}_{\mathrm{H}}(\boldsymbol{\theta})$. Assuming an ellipse shape for the heart contour, we have

$$
\mathbf{C}_{\mathrm{H}}(\boldsymbol{\theta})=\left\{(x, y): \boldsymbol{\theta}^{T} \mathbf{v}=0 ; \quad 4 a c-b^{2}>0\right\}
$$

where the parameter vector $\boldsymbol{\theta}=\left[\begin{array}{llllll}a & b & c & d & e & f\end{array}\right]^{T}$ and $\mathbf{v}=$ $\left[\begin{array}{llllll}x^{2} & x y & y^{2} & x & y & 1\end{array}\right]^{T}$. The last term $J_{4}(\mathbf{C})$ enforces the smoothness (the local properties) of the contour. 
When actually minimizing the energy functional (1), we adopt a level set approach [8] where the contour $\mathbf{C}$ is embedded as the zero level set of a function $\phi(x, y)$, i.e.,

$$
\mathbf{C}=\{(x, y) \in \Omega: \phi(x, y)=0\},
$$

where $\Omega$ defines the image domain. Hence, $J(\mathbf{C})$ becomes $J(\phi)$ and we have an energy functional

$$
\begin{aligned}
J(\phi)= & \lambda_{1} J_{1}(\phi)+\lambda_{2} J_{2}(\phi)+\lambda_{3} J_{3}(\phi)+\lambda_{4} J_{4}(\phi) \\
= & \int_{\Omega} \lambda_{1} M_{1}(x, y) \mathcal{H}_{\epsilon}(\phi)+\lambda_{1} M_{2}(x, y)\left[1-\mathcal{H}_{\epsilon}(\phi)\right] \\
& \quad+P(x, y) \delta_{\epsilon}(\phi)\|\nabla \phi\| d x d y,
\end{aligned}
$$

where

$$
M_{k}=\frac{1}{2} \ln \left(2 \pi \sigma_{k}^{2}\right)+\frac{\left(u(x, y)-m_{k}\right)^{2}}{2 \sigma_{k}^{2}} \quad \text { for } \quad k=1,2
$$

are the negative log of the probability density functions of the object and the background models, respectively; $\mathcal{H}_{\epsilon}$ and $\delta_{\epsilon}$ are the regularized Heaviside and delta functions used to mask the pixels inside and on the contour $\mathbf{C}$, respectively; and the potential function

$$
P(x, y)=\lambda_{2} \Upsilon(x, y)+\lambda_{3} \mathcal{D}^{2}(x, y)+\lambda_{4},
$$

where $\Upsilon$ is the edge map [3] derived from the image, and $\mathcal{D}=\boldsymbol{\theta}^{T} \mathbf{v}$ represents the signed distance to the ellipse contour of the heart.

We minimize the functional (4) by iterating between three tasks. In the first and second tasks, we fix the contour $\mathbf{C}$, and then estimate the parameters $m_{k}$ and $\sigma_{k}^{2}$ for $k=1,2$ of both models in $J_{1}(\phi)$ and the parameters $\boldsymbol{\theta}$ of the ellipse contour in $J_{3}(\phi)$. In the third task, we fix all these parameters, and then evolve the contour $\mathbf{C}$, or equivalently the level set function $\phi$, according to the contour evolution equation

$$
\frac{\partial \phi}{\partial t}=\left[\lambda_{1}\left(M_{1}-M_{2}\right)-\nabla P \cdot \frac{\nabla \phi}{\|\nabla \phi\|}-P \kappa\right] \delta_{\epsilon}(\phi) .
$$

where $\cdot$ is the vector dot product; $\nabla$ is the gradient operator;

$$
\kappa=\operatorname{div}\left(\frac{\nabla \phi}{\|\nabla \phi\|}\right)
$$

representing the curvature along the contour $\mathbf{C}$; and the potential force field

$$
\nabla P=\lambda_{2} \nabla \Upsilon+2 \lambda_{3} \mathcal{D} \nabla \mathcal{D} .
$$

Details on the formulation of the energy functional (4), the derivation of the partial differential equation for the contour evolution (7), and the estimations of all parameters can be found in [6,7].

\section{2. $\lambda$-Annealing}

The energy functional (1) has four terms. Balancing these terms poses very difficult challenges in automatic image segmentation. We learn from our experience with real cardiac MR data that these different terms should be given different weights as the segmentation progresses. For example, at the beginning of the segmentation process, we should first let an arbitrarily placed contour to freely evolve (under the image conditions) toward desired boundary of the object (the left or right ventricle). Hence, the first and second terms, $J_{1}$ and $J_{2}$, should initially play a dominant role because they are derived from the image. However, as the segmentation progresses, the shape prior $J_{3}$ should weigh more heavily, so the final contour resembles, as much as possible, the desired ellipse shape. Finally, the contour smoothness term $J_{4}$ should be enforced constantly throughout the segmentation process. This dynamic adaptation of the functional (1) can be achieved by annealing the relative weights $\lambda_{k}$ for $k=1,2,3,4$, so that $\lambda_{1}$ and $\lambda_{2}$ start high and end low, while $\lambda_{3}$ goes from low value to high value, and $\lambda_{4}$ stays constant.

Our segmentation algorithm nests two loops: the inner loop minimizes the energy functional (4) by iterating between the three tasks described in 2.1, and the outer loop sets each $\lambda_{k}$ for $k=$ $1,2,3$ according to the corresponding annealing schedules below:

$$
\begin{aligned}
& \lambda_{1}(n)=\lambda_{1}^{\mathrm{i}}-\frac{n\left(\lambda_{1}^{\mathrm{i}}-\lambda_{1}^{\mathrm{f}}\right)}{N}, \\
& \lambda_{2}(n)=\frac{1}{2}\left(\lambda_{2}^{\mathrm{i}}-\lambda_{2}^{\mathrm{f}}\right)\left(1+\cos \left(\frac{n \pi}{N}\right)\right)+\lambda_{2}^{\mathrm{f}}, \\
& \lambda_{3}(n)=\frac{\lambda_{3}^{\mathrm{f}}-\lambda_{3}^{\mathrm{i}}}{\cosh \left(10\left(\frac{n \pi}{N}-1\right)\right)}+\lambda_{3}^{\mathrm{i}},
\end{aligned}
$$

where $n$ is the (outter loop) iteration number, $N$ is the total number of (outer loop) iterations, and $\lambda_{k}^{\mathrm{i}}$ and $\lambda_{k}^{\mathrm{f}}$ are the initial and final values of $\lambda_{k}$ for $k=1,2,3$, respectively. The annealing schedule (10) is linearly decreasing the value for $\lambda_{1}$. The annealing schedule (11) reduces $\lambda_{2}$ value moderately in the middle range according to a cosine function. The annealing schedule for $\lambda_{3}$ increases from the initial value $\lambda_{3}^{\mathrm{i}}$ to the final value $\lambda_{3}^{\mathrm{f}}$ most dramatically at the end of the process. Finally, we choose $\lambda_{4}$ to be constant throughout the segmentation process.

\section{RESULTS AND QUALITY ASSESSMENT}

Fig.1(a) depicts the segmentation results obtained automatically by STACS and Fig.1(b) shows the contours traced manually by an expert on the same set of 3 real cardiac MR sequences of a rat, each with 8 frames throughout one cardiac cycle. Three sets of contours, representing the boundaries of the epicardium (the outter contour), the left ventricle (the lower inner contour), and the right ventricle (the upper inner contour) of the rat's heart are present. Although STACS's segmentation results in Fig.1(a) are visually pleasing, we want to find a more quantitative approach to compare our automatically generated contours in Fig. 1(a) with the manually traced "gold standard" contours in Fig. 1(b). We introduce two similarity measures: the area and shape similarity measures.

\subsection{Area Similarity Measure (ASM)}

Let $\mathbf{C}_{a}$ be a set of points on an automatically generated contour, and $\mathbf{C}_{r}$ be a set of points on a reference contour (or the hand-traced contour). One of the methods currently and commonly used in MR image segmentation is comparing the areas within $\mathbf{C}_{a}$ and $\mathbf{C}_{r}$ [9].

$$
S_{\text {area }}=\frac{2 n\left(A_{a} \wedge A_{r}\right)}{n\left(A_{a}\right)+n\left(A_{r}\right)},
$$

where $A_{a}$ and $A_{r}$ are the set of pixels representing the areas within contours $\mathbf{C}_{a}$ and $\mathbf{C}_{r}$, respectively; $n(A)$ represents the number of elements in $A$; and $\wedge$ is the element-wise "and" operator. According to [9], $S_{\text {area }}>0.7$ indicates an excellent agreement between the two comparing regions but the absolute value of $S_{\text {area }}$ may be difficult to interpret. 


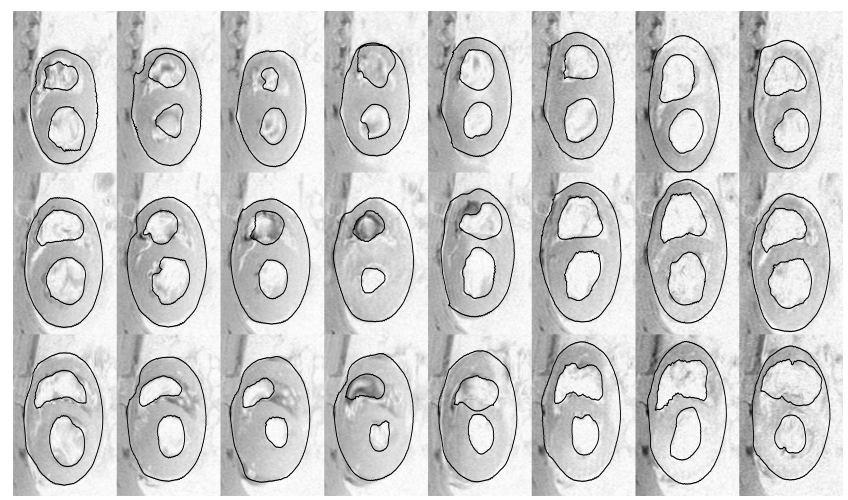

(a) Contours obtained automatically by STACS.

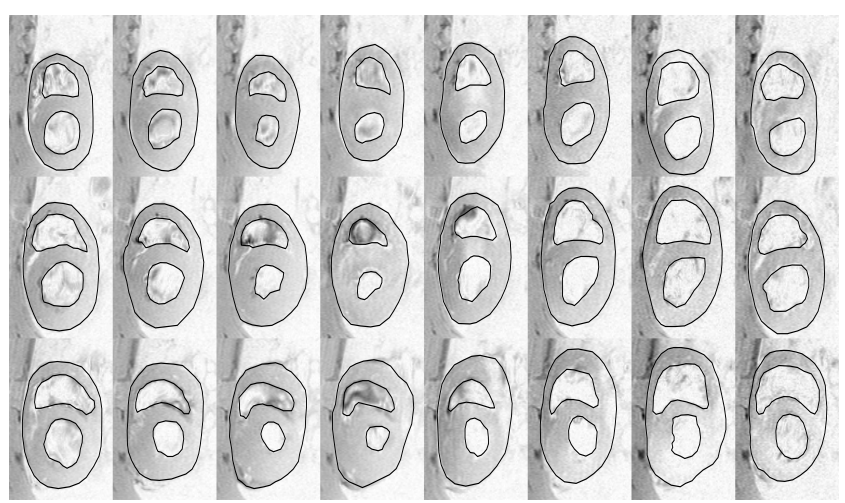

(b) Contours traced manually by an expert.

Fig. 1. Comparing the segmentation results obtained automatically by STACS in (a) with the "gold standard" hand-traced results in (b): three cardiac sequences of eight frames each throughout one cardiac cycle; three contours in each image are the epicardium (outer contour), the left ventricle (inner lower contour), and the right ventricle (inner upper contour).

We computed the area similarity measure (ASM) for the left ventricle (LV), the right ventricle (RV), and the epicardium (EP) contours and summarize their values in three matrices: $S_{\text {area }}^{\mathrm{LV}}, S_{\text {area }}^{\mathrm{RV}}$, and $S_{\text {area }}^{\mathrm{EP}}$, respectively. An entry $(i, j)$ in each of these matrices is the ASM value corresponding to the $(i, j)$ image pair in Fig. 1(a) and (b).

$$
\begin{aligned}
S_{\text {area }}^{\mathrm{LV}} & =\left[\begin{array}{llllllll}
0.94 & 0.74 & 0.85 & 0.77 & 0.85 & 0.94 & 0.89 & 0.92 \\
0.94 & 0.85 & 0.85 & 0.90 & 0.91 & 0.95 & 0.86 & 0.93 \\
0.89 & 0.87 & 0.94 & 0.83 & 0.94 & 0.85 & 0.82 & 0.78
\end{array}\right], \\
S_{\text {area }}^{\mathrm{RV}} & =\left[\begin{array}{llllllll}
0.81 & 0.65 & 0.56 & 0.67 & 0.85 & 0.93 & 0.84 & 0.91 \\
0.91 & 0.75 & 0.74 & 0.82 & 0.79 & 0.93 & 0.87 & 0.90 \\
0.82 & 0.80 & 0.65 & 0.70 & 0.79 & 0.89 & 0.93 & 0.84
\end{array}\right], \\
S_{\text {area }}^{\mathrm{EP}} & =\left[\begin{array}{llllllll}
0.97 & 0.97 & 0.95 & 0.97 & 0.95 & 0.96 & 0.93 & 0.93 \\
0.97 & 0.97 & 0.97 & 0.96 & 0.97 & 0.96 & 0.97 & 0.96 \\
0.95 & 0.95 & 0.96 & 0.96 & 0.96 & 0.96 & 0.96 & 0.96
\end{array}\right] .
\end{aligned}
$$

We observe that all entries of $S_{\text {area }}^{\mathrm{LV}}$ are greater than 0.74 . For the right ventricles, we find 19 out of 24 frames having the ASM values greater than 0.7 , while the remaining 5 values are moderately below (or equal to) 0.7 . We also observe that turbulent blood flow appears in each of the corresponding frames with below 0.7 ASM values. This turbulent blood flow may be the cause for the less satisfactory performance of the segmentation of the right ventricle with these images. Finally, all entries of $S_{\text {area }}^{\mathrm{EP}}$ are above 0.9, indicating excellent result agreement with the gold standard.

\subsection{Shape Similarity Measure (SSM)}

Although ASM, when comparing two contours, is a good indicator on how similar their sizes (or areas) and their relative locations are, it may be less informative with respect to details on the shapes of the two contours. A pair of very different shape contours may yield the same area similarity measure as a pair of contours with identical shape as long as their intersecting regions and the sums of individual areas inside the contour pair are the same. We introduce an alternative to assess the similarity in "shape" between a pair of contours, a modification of the chamfer matching method. Followed the idea described in [10], we modify the chamfer matching method so that it appropriately measures the similarity in shape between two given contours.

Let $\mathbf{C}_{a}$ be a set of points on the contour automatically generated by STACS and $\mathbf{C}_{r}$ be the set of points on the reference contour obtained manually. Our goal is to find a similarity measure $S_{\text {shape }} \in[0,1]$ that quantitatively assesses how similar the shape of the two contours are. Our algorithm for the determination of the shape similarity measure (SSM) proceeds in several steps. First, we generate the binary edge templates, $E_{a}$ and $E_{r}$, where the "on" pixels represent the pixels on each of the two contours being compared. Second, we propagate the shape of the contours in each binary edge template by applying the signed Euclidean distance transform

$$
D(x, y)=\left\{\begin{array}{cl}
-\min _{\mathbf{p} \in \mathbf{C}}\|\mathbf{p}-\mathbf{q}\| & \text { if } \mathbf{q} \text { is inside } \mathbf{C} \\
\min _{\mathbf{p} \in \mathbf{C}}\|\mathbf{p}-\mathbf{q}\| & \text { if } \mathbf{q} \text { is outside } \mathbf{C}
\end{array},\right.
$$

where $\mathbf{q}$ is a pixel in the image domain, $\mathbf{p} \in \mathbf{C}$ represents a pixel on the contour $\mathbf{C}$, and $\|\cdot\|$ is the Euclidean norm. Applying the signed Euclidean distance transform (14) to the binary edge templates $E_{a}$ and $E_{r}$, we obtain the corresponding distance maps, $D_{a}$ and $D_{r}$, respectively. These distance maps simply contain the scaling replicas of the contour shapes throughout the image domain. Third, we calculate the corresponding phase maps by taking the inverse tangent of the ratio of the gradient components in each signed distance map, i.e.,

$$
\Phi(x, y)=\tan ^{-1} \frac{\nabla_{y} D(x, y)}{\nabla_{x} D(x, y)}
$$

where $\nabla_{x} D$ and $\nabla_{y} D$ represent the $x$ and $y$ components of the gradient of the signed distance map $D$, respectively. In the fourth step, we compute the normalized phase similarity between the two contours by

$$
S_{\text {phase }}=\frac{\left|\Phi_{a}-\Phi_{r}-\pi\right|}{\pi},
$$

where $\Phi_{a}$ and $\Phi_{r}$ are the corresponding phase maps of contours $\mathbf{C}_{a}$ and $\mathbf{C}_{r}$, respectively. The index $S_{\text {phase }}$ takes values in $[0,1]$, the value of 1 indicates that the two contours have the same phase and a value of 0 refers to the maximum phase difference of $\pi$. In the final step, we measure the shape similarity by taking the weighted sum of the phase similarity measure along $\mathbf{C}_{a}$ against $\mathbf{C}_{r}$, i.e.,

$$
S_{\text {shape }}=\frac{1}{n\left(\mathbf{C}_{a}\right)} \sum_{(x, y) \in \mathbf{C}_{a}} \Gamma_{r}(x, y) S_{\text {phase }}(x, y),
$$


where $n\left(\mathbf{C}_{a}\right)$ denotes the number of pixels on the contour $\mathbf{C}_{a}$, and $\Gamma_{r}(x, y) \in[0,1]$ is derived from $D_{r}$ as

$$
\Gamma_{r}(x, y)=\exp \left\{-\frac{D_{r}^{2}(x, y)}{\sigma^{2}}\right\}
$$

where $\sigma^{2}$ is a positive constant.

The findings for the SSM of the LV, RV, and the EP contours are respectively the following:

$S_{\text {shape }}^{\mathrm{LV}}=\left[\begin{array}{cccccccc}0.89 & 0.70 & 0.86 & 0.75 & 0.83 & 0.92 & 0.87 & 0.83 \\ 0.91 & 0.77 & 0.77 & 0.91 & 0.84 & 0.91 & 0.70 & 0.89 \\ 0.80 & 0.81 & 0.92 & 0.83 & 0.92 & 0.80 & 0.68 & 0.62\end{array}\right]$,

$S_{\text {shape }}^{\mathrm{RV}}=\left[\begin{array}{llllllll}0.79 & 0.43 & 0.64 & 0.40 & 0.72 & 0.89 & 0.75 & 0.90 \\ 0.88 & 0.73 & 0.70 & 0.83 & 0.65 & 0.89 & 0.78 & 0.87 \\ 0.81 & 0.87 & 0.78 & 0.77 & 0.67 & 0.84 & 0.87 & 0.68\end{array}\right]$,

$S_{\text {shape }}^{\mathrm{EP}}=\left[\begin{array}{cccccccc}0.92 & 0.90 & 0.85 & 0.92 & 0.88 & 0.88 & 0.75 & 0.74 \\ 0.92 & 0.91 & 0.91 & 0.89 & 0.90 & 0.87 & 0.89 & 0.86 \\ 0.87 & 0.84 & 0.90 & 0.86 & 0.87 & 0.89 & 0.86 & 0.91\end{array}\right]$.

For the left ventricles, 21 out of 24 values of the SSM are above 0.7. For the right ventricles, 17 out of 24 values of the SSM are above 0.7 , where $5 \mathrm{SSM}$ values are between 0.6 and 0.7 , and 2 SSM values fall below 0.5. Again, in the image frames with low similarity measures, blood flow may have affected the performance of the algorithm. Finally, all of the SSM values for the epicardial contours are above 0.7 , indicating excellent segmentation agreement with the gold standard.

\section{RESULT ANALYSIS}

The very good visual agreement between the segmentation results by STACS and by human expert is confirmed both by the area similarity measure (ASM) and the shape similarity measure (SSM) shown by the matrices in the previous section. Here we compute average statistics of the ASM and SSM results presented above. The best results are obtained for the epicardium where the average ASM is $0.95 \pm 0.01$ and the average SSM is $0.87 \pm 0.05$. These good results may be attributed to the role played by the shape prior because the epicardium does look visually like an ellipse. Although STACS still segments very well the right ventricle with an average ASM of $0.81 \pm 0.10$, and SSM of $0.75 \pm 0.13$, these numbers are smaller than the average values for the other two contours. This can be attributed to the fact that the shape of the right ventricle is not as close to an ellipse as the left ventricle or the epicardium, and due to the presence in many MR frames of turbulent blood flow. We note that the left ventricle is well segmented, with the average ASM of $0.87 \pm 0.06$ and the average SSM of $0.82 \pm 0.09$, even though the presence of the papillary muscles represents in practice a major challenge in other segmentation algorithms. As may be expected, the SSM generally yields a smaller value than the ASM because the SSM is more sensitive to local discrepancies between the contours than the ASM, which is a more global measure. Finally, we observe that the standard deviations of all average similarity measures are quite small, implying that STACS consistently produces good results.

\section{CONCLUSION}

Automatic segmentation of the heart in MR image sequences of small animals is desired in medical studies because of the enormous amount of data involved. Our studies have been concerned with studying the rejection of heart transplantation in rats and mice [11]. We have presented in this paper a new automatic segmentation algorithm, stochastic active contour scheme (STACS), and showed with several real cardiac MR data very good agreement between STACS results and the "gold standard" provided by an expert segmenting the same images. The three main features of STACS are: stochastic modeling of the textures of the different anatomy structures and the heart muscle; the incorporation of the heart shape prior; and the annealing schedules on the parameters weighing the contribution of each of the four terms in the energy functional. The good quality of the segmentation results by STACS are confirmed not only visually but also quantitatively by computing two similarity measures, area and shape. The average values of both measures are above 0.7 for the epicardium, the left ventricle, and the right ventricle.

\section{REFERENCES}

[1] M. Kass, A. Witkin, and D. Terzopoulos, "Snakes: Active contour models," Internatinal Journal of Computer Vision, vol. 1, no. 4, pp. 321-331, 1988.

[2] R. Malladi, J. A. Sethian, and B. Vemuri, "Shape modeling with front propagation: A level set approach," IEEE Transactions on Pattern Analysis and Machine Intelligence, vol. 17, no. 2, pp. 158-175, February 1995.

[3] C. Xu and J. L. Prince, "Snakes, shapes, and gradient vector flow," IEEE Transactions on Medical Imaging, vol. 7, pp. 359-369, March 1998.

[4] T. F. Chan and L. A. Vese, "Active contours without edges," IEEE Transactions on Image Processing, vol. 10, no. 2, pp. 266-277, Febuary 2001.

[5] A. Yezzi Jr., A. Tsai, and A. Willsky, "A statistical approach to snakes for bimodal and trimodal imagery," in The Proceedings of the Seventh IEEE International Conference on Computer Vision, 1999, vol. 2, pp. 898 -903.

[6] Charnchai Pluempitiwiriyawej, José M. F. Moura, Yi-Jen Lin Wu, Shinichi Kanno, and Chien Ho, "Stochastic active contour for cardiac MR image segmentation," in IEEE International Conference on Image Processing (ICIP) Barcelona, Spain, September 2003.

[7] Charnchai Pluempitiwiriyawej, José M. F. Moura, Yi-Jen Lin Wu, Shinichi Kanno, and Chien Ho, "STACS: New active contour scheme for cardiac MR image segmentation," submitted for publication, November 15, 2003. 25 pages.

[8] J. A. Sethian, Level Set Methods and Fast Marching Methods, Cambridge University Press, 1999.

[9] M. Stella Atkins and Blair T. Mackiewich, "Fully automatic segmentation of the brain in MRI," IEEE Transactions on Medical Imaging, vol. 17, no. 1, pp. 98-107, Febuary 1998.

[10] Jia-Ching Cheng and José M. F. Moura, "Capture and representation of human motion in live video sequences," IEEE Transactions on Multimedia, vol. 1, no. 2, pp. 144-156, June 1999.

[11] Yi-Jen Lin Wu, Shinichi Kanno, and Chien Ho, "Noninvasive early detection of rejection for transplanted hearts with multi-dimensional cardiac MRI," Journal of Cardiovascular Magnetic Resonace, vol. 4, no. 1, pp. 4-6, 2002. 